\title{
Resonant enhancement of grazing incidence neutron scattering for the characterization of thin films
}

\author{
Adrien Perrichon $\odot,{ }^{1,2, *}$ Anton Devishvili $\odot,{ }^{1,3}$ Kristina Komander, ${ }^{1}$ Gunnar K. Pálsson $\odot,{ }^{1}$ Alexei Vorobiev, ${ }^{1,3}$ \\ Rasmus Lavén, ${ }^{4}$ Maths Karlsson ${ }^{0},{ }^{4}$ and Max Wolff ${ }^{1, \dagger}$ \\ ${ }^{1}$ Department of Physics and Astronomy, Uppsala University, SE-752 37 Uppsala, Sweden \\ ${ }^{2}$ ISIS Facility, STFC Rutherford Appleton Laboratory, Didcot OX11 OQX, United Kingdom \\ ${ }^{3}$ Institut Laue-Langevin, 71 avenue des Martyrs, 38000 Grenoble, France \\ ${ }^{4}$ Department of Chemistry and Chemical Engineering, Chalmers University of Technology, SE-412 96 Göteborg, Sweden
}

(Received 26 January 2021; revised 19 April 2021; accepted 28 May 2021; published 17 June 2021)

\begin{abstract}
We use signal enhancement in a quantum resonator for the characterization of a thin layer of vanadium hydride using neutron reflectometry and demonstrate that pressure-concentration isotherms and expansion coefficients can be extracted from the measurement of totally externally reflected neutrons only. Moreover, a consistent data analysis of the attenuation cross section allows us to detect and quantify off-specular and small angle scattering. As our experiments are effective direct beam measurements, combined with resonant signal enhancement, counting times become considerably reduced. This allows us to overcome the challenges resulting from the comparatively low brilliance of neutron beams for grazing incidence scattering experiments. Further, we discuss the potential of resonant enhancement to increase any scattering, which is of particular interest for grazing incidence small angle neutron scattering and spectroscopy.
\end{abstract}

DOI: 10.1103/PhysRevB.103.235423

\section{INTRODUCTION}

Over the last decades surface science has advanced continuously and considerably. This is related to the increased interest in surfaces and interfaces among the scientific community and their importance for many technological applications. The understanding of surface and interfacial properties of materials as well as interfacial processes in terms of structural, morphological, and compositional changes is of crucial importance in many scientific fields that include chemistry, physics, biology, and engineering.

Driven by the need to characterize surfaces and interfaces, experimental methods and probes have been developed and improved to enter previously inaccessible territories. For instance, the advancements of atomic force [1] and electron [2] microscopes resulted in totally new opportunities for science and technology. The developments of both methods resulted in compact and readily available instruments, which are now used routinely. Another example is the development of photoelectron spectroscopy [3] or surface x-ray scattering in general. With the development of synchrotron $\mathrm{x}$-ray sources providing flexible wavelength and high brilliance, previously unachieved sensitivity was reached for

\footnotetext{
*adrien.perrichon@stfc.ac.uk

${ }^{\dagger}$ max.wolff@physics.uu.se
}

Published by the American Physical Society under the terms of the Creative Commons Attribution 4.0 International license. Further distribution of this work must maintain attribution to the author(s) and the published article's title, journal citation, and DOI. Funded by Bibsam. compositional and structural studies of surfaces. For instance, the electron density across interfaces can be extracted with Ångström resolution from the specular (incident beam angle equals exiting beam angle) reflectivity of $x$ rays. Further, offspecular or grazing incidence scattering provides information about in-plane ordering and fluctuations, and spectroscopy provides element specific information.

In reflectivity measurements, the incident beam impinges onto a sample surface under a shallow angle and optical effects, e.g., total external reflection, have to be taken into account. At the critical wave vector for total external reflection, the incident and reflected wave fields are in phase at an interface, which amplifies scattering from impurities or roughness [4]. For more complex structures, like single [5] or multilayers [6], scattering is amplified whenever a standing wave field develops, which is referred to as resonant enhanced or resonant diffuse scattering. Theoretically, the scattering from rough surfaces can be described in the framework of a first order perturbation theory, the distorted wave Born approximation, as described in Ref. [7], which was later extended to layered structures [8]. For resonance effects at very small momentum transfers, it may happen that scattering from the evanescent wave field leads to intensity missing from the totally externally reflected signal, which shows up as dips in the total reflection region $[9,10]$.

However, sensing light element or magnetism in buried interfaces is challenging by using $\mathrm{x}$ rays. Still, such materials are highly important, and many scientific questions are not yet resolved. Examples of such questions are the dynamics of lithium [11] and protons [12] in thin films for battery or fuel cell applications, and biology [13], or the diffusivity of water in cell membranes [14], proteins, or micelles [15]. One other area of research which is highly relevant for technology 
and the development of new materials is the understanding of surface dynamics in polymers and glasses [16-18].

Compared to electrons and photons, neutrons are sensitive to light elements in the presence of heavy ones and can penetrate deep into matter, as they interact with the nuclei and allow the study of slow dynamics. Moreover, the sensitivity to the nucleus allows contrast variation experiments by isotope substitution. The drawback of neutron scattering methods, however, is that they are only available at large scale facilities and that the brilliance of nowadays neutron sources is limited. As a result, many neutron scattering studies are flux limited, an issue that is particularly relevant for small sample volumes or surface scattering studies.

The experimental method in neutron scattering providing information about interfaces is neutron reflectometry, the equivalent to $\mathrm{x}$-ray reflectometry. In the case of neutrons, it is the density profile of the isotopes across an interface that is extracted from the specularly reflected intensity. For recent reviews on neutron reflectivity see Refs. $[19,20]$. As for $\mathrm{x}$ rays, lateral fluctuations result in additional scattering, which is known as off-specular or grazing incidence scattering [21].

The signal scattered from a thin film can become significantly enhanced and the sensitivity of neutron studies can be improved if resonant effects are used. Pfeiffer $e t$ al. measured the wave field in a neutron resonator emitted along the sample horizon and showed that effectively a coherent microbeam of neutrons can be generated by this method [22]. The enhancement factor for scattering and absorption experiments using neutron resonators was calculated by Radu et al. [23] for multilayer systems. Over the years, resonators have been applied to enhance the absorption and subsequent emission of gamma radiation [24] or alpha particles [25] in order to improve the depth sensitivity of neutron reflection measurements, as well as amplify the surface scattering signal in diffraction [26]. The magnetic moment of the neutron allows us to probe the magnetic induction in layered structures. In this context resonant enhancement was used for the study of magnetic domains [27], noncollinearity [28], as well as the proximity effect at the ferromagnet-superconductor interface [29]. A review on the use of planar neutron waveguides can be found in Ref. [30]. Moreover, it was shown that the incoherent scattering from hydrogen in a thin layer of vanadium can be detected, which brings into reach inelastic and quasielastic neutron scattering studies of the dynamics in thin films [31]. Following along the same line the interfacial dynamics of a bicontinuous polymer in a sponge phase was revealed by using a resonant enhancement in the adjacent substrate $[32,33]$.

In the present work we use polarized neutron reflectometry to characterize a quantum resonator thin film. The absorption and scattering from the thin film at the resonance conditions appear as intensity missing from the totally externally reflected neutrons, i.e., as dips in the reflectivity profile. The momentum transfer and missing intensity associated to these dips can be connected to the composition and thickness of the resonating layer. In particular, the missing intensity is very sensitive to any scattering, coherent as well as incoherent. This opens opportunities to obtain information on, for instance, the thin film composition and thickness by measuring the total external reflection region only, which, because of the high intensity of the incident and reflected beams, allows for

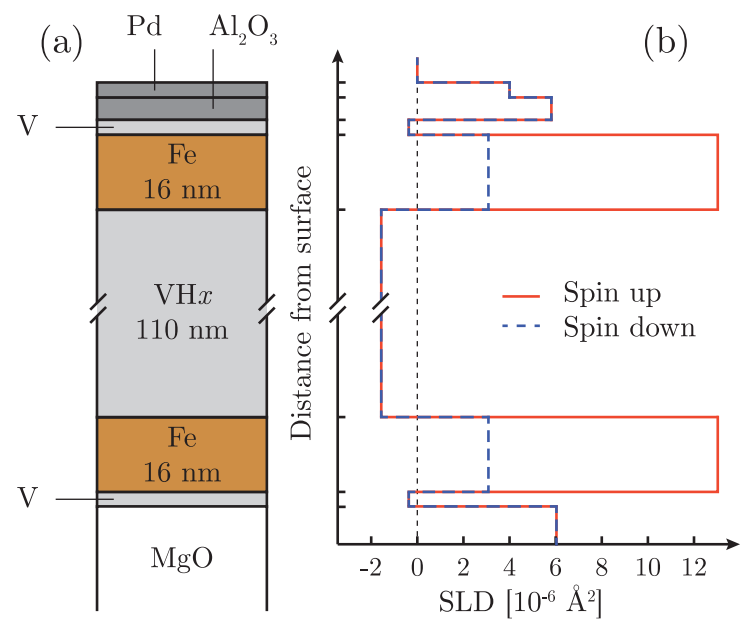

FIG. 1. (a) Scheme of the structure of the vanadium hydride resonator thin film. (b) Spin up (red line) and spin down (blue dashed line) scattering length density (SLD) profiles of the sample. Dimensions are not to scale.

short counting times, thus faster experiments. This is critical in particular for time-resolved or in situ parametric studies, where the composition or thickness of the layer changes as a function of external parameters, such as temperature, gas exposure, or magnetic field.

As the model system for our studies, we have chosen a thin film of vanadium hydride, a system which scatters mainly incoherently. This system offers the possibility to build neutron resonators in a straightforward way [31], and the bulk properties have been studied extensively with neutron scattering methods during the 70's [34]. More recently, it was shown that the thermodynamics [35] and diffusivity [36] of hydrogen in thin vanadium films drastically changes with respect to bulk. However, the optical methods used in these studies are indirect and a verification of the results with neutrons being directly sensitive to the hydrogen is strongly desirable.

The aim of the study is to quantify the hydrogen content inside the vanadium hydride thin film as a function of applied hydrogen pressure, which allows us to extract pressure-concentration isotherms and expansion coefficients. To improve the robustness of our approach we combine the quantum resonator with magnetic contrast variation [37] to tune the height of the potential well of the resonator. Our results show that information of the composition of the model vanadium hydride resonator thin film can be reliably extracted from the region of total external reflection.

\section{METHODS}

\section{A. Sample}

The scheme of the vanadium hydride resonator thin film is shown in Fig. 1. The sample was grown by magnetron sputtering on a $\mathrm{MgO}$ substrate, following an earlier protocol for the growths of vanadium films [38,39]. It consists of a $\sim 110 \mathrm{~nm}$ thick film of vanadium confined between two iron layers with thicknesses of $\sim 16 \mathrm{~nm}$. Vanadium is known to readily absorb hydrogen while iron does not at the conditions of our experiments. Indeed, since the hydrogen concentration 
depends exponentially on the enthalpy of absorption, which takes values of $-0.30 \mathrm{eV}$ and $+0.29 \mathrm{eV}$ per hydrogen atom for hydrogen in vanadium [40] and iron [41], respectively, the hydrogen concentration in the iron layer can be completely neglected in the present pressure and temperature range. The transport of hydrogen to the vanadium layer is insured by the large diffusivity and low activation energy of hydrogen permeation in high-purity iron [42]. A capping layer of $\mathrm{Al}_{2} \mathrm{O}_{3}$ prevents oxidation of the vanadium layer, and an outermost palladium layer facilitates the dissociation of hydrogen. Two monolayers of vanadium are used as seed layers to ensure the growth of the following layers [38,39]. The sample was characterized by $\mathrm{x}$-ray and neutron reflectometry in a previous study and displays the predicted dips in the total external reflection region [31].

\section{B. Neutron reflectometry}

Polarized neutron reflectometry has been performed at SuperADAM $[43,44]$ at the Institut Laue Langevin (Grenoble, France). A fixed neutron wavelength of $\lambda=5.225 \AA$ with incident polarization of $99.7 \%$ has been selected by a highly oriented pyrolytic graphite (HOPG) monochromator with double band pass mirror polarizer. The incident collimation for this experiment was $0.16 \mathrm{mrad}$. Taking into account $\Delta \lambda / \lambda=0.5 \%$, this results in a resolution in momentum transfer $Q_{z}$ of $\Delta Q_{z}=1.2 \times 10^{-4} \AA^{-1}$, which is sufficient to resolve the dips resulting from the quantum resonator in the region where total external reflection occurs.

In contrast with our previous experiment on this system [31], the polarization of the reflected signal is here not analyzed, as it is not required for the purpose of the measurement. It follows that only two reflectivity profiles are measured, corresponding to spin up and spin down polarized neutrons.

An ultrahigh vacuum (UHV) chamber with windows for the neutron beam was used to apply a well-defined pressure of hydrogen to the sample. Baking of the sample chamber resulted in a base pressure below $\sim 10^{-8}$ mbar prior to exposure to hydrogen gas. A set of manual valves with external connectors allowed us to introduce hydrogen from a pressurized hydrogen bottle into the chamber. To eliminate impurities below ppb level the hydrogen was filtered through a NuPure purifier. The temperature was controlled by a Lakeshore temperature controller connected to an external heating jacket minimizing thermal gradients. The temperature of the sample was kept at $473 \mathrm{~K}$.

In order to maintain the polarization of the neutrons over the large sample environment, a magnetic field of about 30 Oe was applied using a large coil encompassing the sample chamber. Note, however, that this field did not allow us to saturate the magnetic moments of the iron layers.

\section{Fit of the reflectivity profiles}

The measured reflectivity profiles were fitted using an in-house developed software [45-47]. For each layer, the thickness and roughness, the real, imaginary, and magnetic part of the SLD (here denoted $\mathrm{SLD}_{\text {real }}, \mathrm{SLD}_{\mathrm{im}}$, and $\mathrm{SLD}_{\mathrm{mag}}$ ), and a scaling factor to the magnetic SLD were included in the model. The starting values of the fits are reported in Table I.
TABLE I. Starting values of the fit of the reflectivity profiles, except $(*)$ the thickness of the $\mathrm{VH}_{x}$ layer evaluated with starting values in the range $1100-1200 \AA$ and $(* *)$ the $\mathrm{SLD}_{\text {real }}$ of the $\mathrm{VH}_{x}$ layer with values in the range $-0.20 \times 10^{-6}$ to $-2.20 \times 10^{-6} \AA^{-2}$.

\begin{tabular}{lcccc}
\hline \hline Layer & $\begin{array}{c}\text { Thickness } \\
(\AA)\end{array}$ & $\begin{array}{c}\mathrm{SLD}_{\text {real }} \\
\left(10^{-6} \AA^{-2}\right)\end{array}$ & $\begin{array}{c}\mathrm{SLD}_{\text {im }} \\
\left(10^{-6} \AA^{-2}\right)\end{array}$ & $\begin{array}{c}\mathrm{SLD}_{\text {mag }} \\
\left(10^{-6} \AA^{-2}\right)\end{array}$ \\
\hline $\mathrm{Pd}$ & 13.0 & 3.98 & $1.3 \times 10^{-3}$ & 0 \\
$\mathrm{Al}_{2} \mathrm{O}_{3}$ & 70.3 & 5.67 & $3.0 \times 10^{-5}$ & 0 \\
$\mathrm{~V}$ & 7.5 & -0.31 & $1.0 \times 10^{-3}$ & 0 \\
$\mathrm{Fe}$ & 157 & 8.02 & $6.0 \times 10^{-4}$ & 5.12 \\
$\mathrm{VH}_{x}$ & $1100(*)$ & $-0.60(* *)$ & $5.0 \times 10^{-3}$ & 0 \\
$\mathrm{Fe}$ & 157 & 8.02 & $6.0 \times 10^{-4}$ & 5.12 \\
$\mathrm{~V}$ & 7.5 & -0.31 & $1.0 \times 10^{-3}$ & 0 \\
$\mathrm{MgO}$ & $\infty$ & 5.98 & $9.4 \times 10^{-6}$ & 0 \\
\hline \hline
\end{tabular}

As further explained in the results section, the reflectivity profiles are fitted over two regions: in the $Q_{z}$ range of $0.004 \leqslant Q_{z} \leqslant 0.025 \AA^{-1}$ and in the $Q_{z}$ range of $0.004 \leqslant$ $Q_{z} \leqslant 0.017 \AA^{-1}$, which will be referred to as "extended" and "pre-edge" regions in the discussion. Note that both regions have limited $Q$ range compared to a standard full reflectivity profile, which is required to accurately determine all the parameters characterizing the sample, which we have measured in a previous study [31]. Because of said limited $Q$ range, the roughness of all layers converges to zero and is kept fixed. Similarly, most parameters that do not significantly impact the reflectivity profiles are kept fixed to the values determined in the previous study [31]. Free parameters are, for the extended region, the thickness, $S L D_{\text {real }}$ and $S L D_{\text {im }}$ of the $\mathrm{VH}_{x}$ layer, the $\mathrm{SLD}_{\text {real }}$ of the Fe layers, and a scaling factor to the magnetic SLD. For the pre-edge region, free parameters are only the thickness, $\mathrm{SLD}_{\text {real }}$ and $\mathrm{SLD}_{\text {im }}$ of the $\mathrm{VH}_{x}$ layer.

\section{Theoretical description of the quantum resonator}

In order to give a qualitative description of the positions of the dips in the total reflectivity resulting from the absorption and scattering inside a neutron resonator, we use the quantum mechanical textbook model system of a particle (the neutron) in a box with infinite walls (infinite SLD). Note that this is a toy model intended to highlight the relationship between the positions of the resonances and the parameters associated with the thin film, such as the thickness of the layer and its SLD. For more detailed calculations of resonant enhancement, including of the signal amplification, we refer to literature $[23,45]$.

The momenta for resonances in a quantum mechanical resonator can be calculated from the following relation:

$$
k_{i, z}=\frac{m \pi}{d},
$$

where $k_{i, z}=k_{i} \sin \alpha_{i}=\frac{2 \pi}{\lambda} \sin \alpha_{i}$ is the component of the neutron wave vector along the $z$ axis, parallel to the surface normal, $m$ is the order of the resonance, and $d$ is the thickness of the resonator. For small incident angles the sinus function can be Taylor expanded and we can calculate the angle and wavelength for the resonances, depending on whether the 

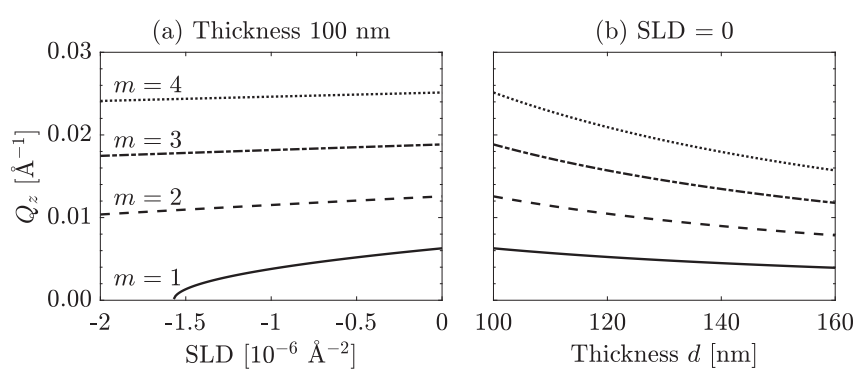

FIG. 2. Position of the first four resonances, $m=1-4$, in $Q_{z}$ depending on (a) the SLD with respect to vacuum and (b) the thickness of the resonator.

experiments are done on an angle dispersive or time-of-flight instrument, respectively:

$$
\begin{aligned}
\lambda_{m} & =\frac{2 d}{m} \sin \alpha_{i}, \\
\alpha_{i, m} & =\arcsin \frac{m \lambda}{2 d} .
\end{aligned}
$$

In the above equations we have not considered refraction effects, which occur if the SLD of the resonator and the material from which the incident beam enters the sample are different, which is usually the case. Taking refraction into account Eq. (3) becomes:

$$
\alpha_{i, m}=\arccos \sqrt{n^{2}-\left(\frac{m \lambda}{2 d}\right)^{2}},
$$

with $n=\sqrt{1-\frac{\lambda^{2} \mathrm{SLD}}{2 \pi}}$ the refractive index for neutrons, which is close to one.

Alternatively, the positions of the resonances can be expressed in terms of the momentum transfer along the surface normal:

$$
Q_{z}=\frac{4 \pi}{\lambda} \sqrt{1-n^{2}+\left(\frac{m \lambda}{2 d}\right)^{2}},
$$

which develops in:

$$
Q_{z}=2 \sqrt{2 \pi \operatorname{SLD}+\pi^{2} \frac{m^{2}}{d^{2}}},
$$

which clearly shows that the positions of the resonance $Q_{z}$ depend on both the SLD and the thickness $d$. This is further shown in Fig. 2, where $Q_{z}$ of the first four resonances, $m=1-$ 4, is calculated according to Eq. (6) and in dependency of the SLD (left panel) and thickness (right panel). While Eq. (6) always has solutions for SLD $\geqslant 0$, in the case where SLD < 0 , the solutions are real numbers only if:

$$
d \leqslant \frac{m \sqrt{\pi}}{\sqrt{-2 \mathrm{SLD}}} .
$$

Since the SLD and the thickness of the resonator both influence its position in $Q_{z}$, this has to be taken into account for investigation of films for which both quantities change at the same time. This happens for many studies that are relevant in neutron reflectometry experiments, like, e.g., hydrogen loading in metals or swelling of organic layers. In magnetism the layer thickness typically remains constant, and the magnetization can be extracted directly from the positions of the resonances. However, one may note that the above calculations assume an infinite height of the resonator walls, which does not hold in real experiments. Accordingly, the resonator thickness calculated by Eq. (6) will overestimate the width of the resonator.

Below the critical angle of total external reflection, "dips" in the reflectivity are visible if signal is missing from the reflected beam. This signal can either be transmitted, absorbed, or scattered. The total flux must be conserved, and we can write:

$$
I_{0}=R+T r+A+S,
$$

with $I_{0}, R, T r, A, S$ the incident beam, reflected, transmitted, absorbed, and scattered intensities, respectively. Below the critical momentum for total external reflection, the transmitted intensity is zero, $T r=0$. It follows that any intensity missing from the reflected beam must be either absorbed or scattered. For neutrons, the scattering can be coherent or incoherent. The incoherent scattering is isotropic and can therefore, for the purpose of reflectivity measurements, be treated in the same way as absorption. Coherent scattering can be Bragg scattering from crystal planes, small angle neutron scattering (SANS), or off-specular scattering from density fluctuations or magnetic domains. The long wavelength used on reflectometers to probe small $Q$ values is typically above the Bragg cutoff for most materials, which means that Bragg scattering can be neglected. On the other hand, SANS or off-specular scattering may be present for films with in-plane fluctuations of the SLD [21].

Considering the above discussion, the depth of the dips in the totally reflected intensity can be calculated as follows:

$$
I_{m}=N\left(4 \pi\left(\Sigma_{\mathrm{abs}}+\Sigma_{\mathrm{inc}}\right)+S_{\mathrm{lowQ}}\right),
$$

with $\Sigma_{\text {inc }}$ the incoherent scattering cross section, $\Sigma_{\text {abs }}$ the absorption cross section, $S_{\text {lowQ }}$ the scattering from fluctuations in the SLD, and $N$ a factor accounting for the wave-field enhancement in the resonator. $\Sigma_{\text {inc }}$ and $\Sigma_{\text {abs }}$ are calculated from the tabulated nuclear cross sections and therefore the depth of the dips provides a measurement of concentrations, which is independent of the positions of the resonances and only related to absorption and incoherent scattering, if no scattering to low $Q$ is present. In turn the scattering towards low $Q$ can be quantified via the signal still missing from the reflected beam after considering the incoherent scattering and absorption.

\section{RESULTS}

We have measured the spin up and spin down reflectivity profiles of the vanadium hydride resonator thin film at $T=473 \mathrm{~K}$ and at different hydrogen pressures. Figure 3(a) depicts the spin up signal measured on the position sensitive detector (PSD) for the lowest pressure data, for which the vanadium layer is assumed free of hydrogen. The region of interest (ROI) from which the specular line is extracted is marked [A]. Note that a significant amount of scattering is present outside of the specular ROI, in the arbitrarily defined ROI marked [B], which then represents nonspecular signal. 
(a)

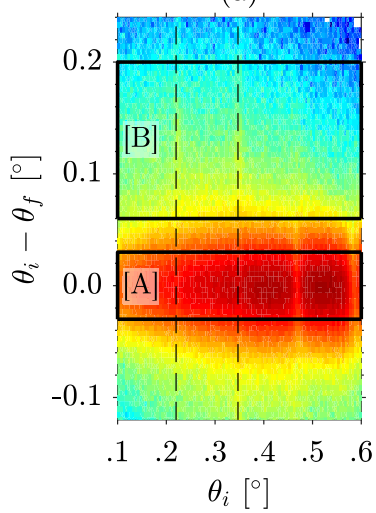

(b)

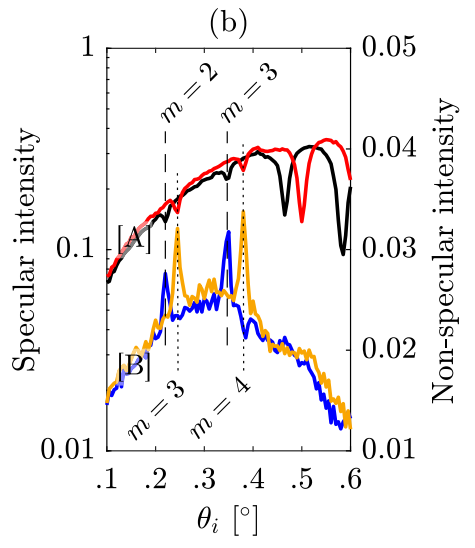

FIG. 3. (a) Color map of the spin up intensity measured on the PSD at $T=473 \mathrm{~K}$ for the lowest pressure data. The ROIs of integration are marked by black rectangles: [A] specular ROI, and [B] nonspecular ROI. The positions of the resonances are marked by vertical dashed lines. (b) Integrated spin up intensities: (black line) specular ROI for lowest pressure data, (blue line) nonspecular ROI for lowest pressure data, (red line) specular ROI for $P \approx 200 \mathrm{mbar}$ data, (orange) nonspecular ROI for $P \approx 200$ mbar data. The positions of the resonances for the lowest pressure data and for the $P \approx$ 200 mbar data are marked by vertical dashed lines and vertical dotted lines, respectively. The order of the resonances is also indicated.

In Fig. 3(b) we show the integrated spin up intensity over the specular and nonspecular ROIs. It is clear by comparing the specular (black line) and nonspecular (blue line) intensities that, at the positions of the resonances (vertical dashed lines), diffuse scattering is present, which appears as sharp peaks in the nonspecular channel. This amplified nonspecular signal, in resonance condition, has been referred to as generalized Yoneda wings [7,8].

Note that these peaks in the nonspecular ROI are on top of a large background. Figure 3(b) also shows the spin up specular (red line) and nonspecular (orange line) intensities for the $\sim 200$ mbar data, where a significant amount of hydrogen is expected to be present in the vanadium hydride layer. The large background in the nonspecular channel is essentially identical for the lowest pressure data and for the $\sim 200$ mbar data, which indicates that it originates from effects independent of the hydrogen content. This broad signal could then originate from blooming effect on the detector, to some extent from low- $Q$ scattering from the substrate due to overillumination of the thin film, or potentially from low- $Q$ scattering from the vanadium (excluding hydrogen) and iron layers, for instance off-specular magnetic scattering from the unsaturated iron layers.

Conversely, the position and intensity of the peaks in the nonspecular channel, which are associated to diffuse scattering from the sample and specifically from the vanadium hydride layer, clearly depend on the hydrogen pressure, hence on the hydrogen content. This is further shown in Fig. 4 that shows the specular reflected intensity of spin up (left panel) and spin down (right panel) polarized neutrons for all measured pressure points. There is a clear shift of the positions of the resonances, marked $m=2-4$, toward lower $Q_{z}$ values, with increasing hydrogen content, as is expected from the model discussed in Sec. II D.

To further describe the data we distinguish two regions in the reflectivity profiles: the region of total external reflection (hereafter referred to as "pre-edge"), for $Q_{z} \leqslant 0.017 \AA^{-1}$, and the region where the beam penetrates the sample, above this value. In the pre-edge region, the dips associated to the resonances are well visible in both spin up and spin down profiles. Above $Q_{z}=0.017 \AA^{-1}$, the interference fringes resulting from beams partially reflected at the different interfaces are well visible, in particular where a large contrast between the iron and vanadium is present for the up polarized neutrons [Fig. 4(b)].

We have performed a fit of the reflectivity profiles over the pre-edge and extended regions. The results of the fit are
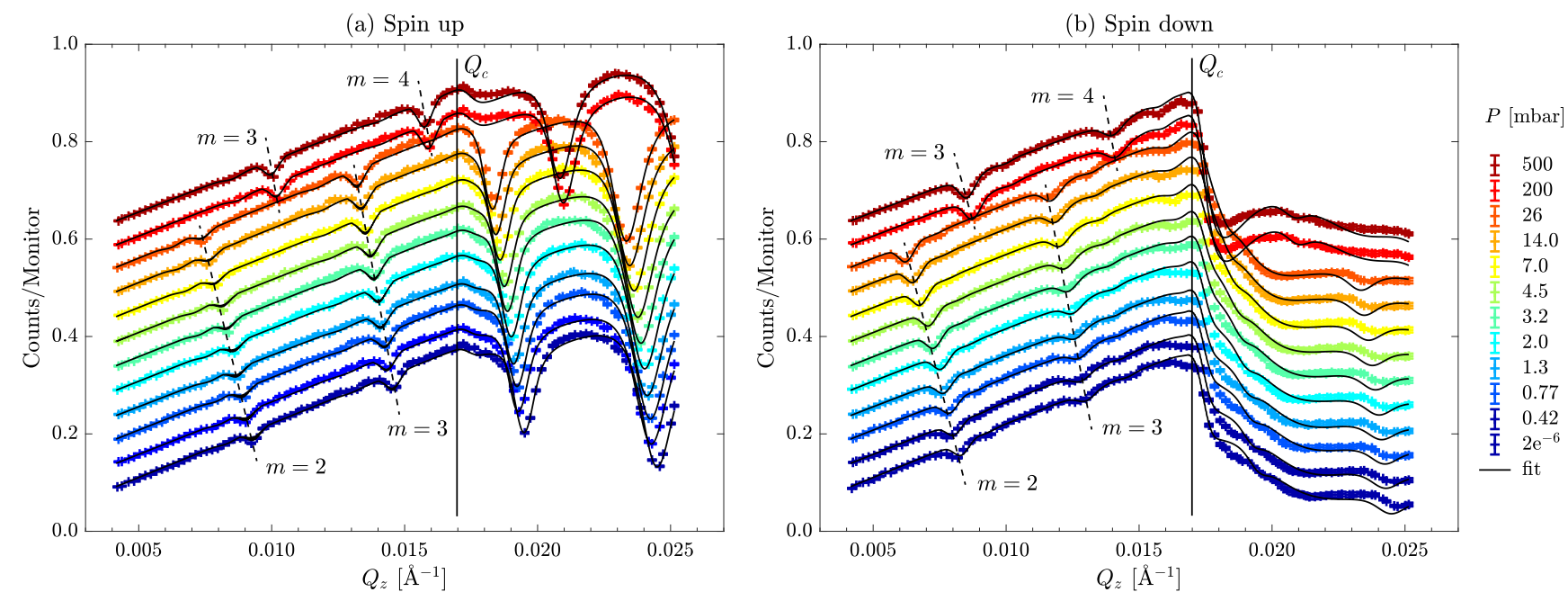

FIG. 4. Reflected intensity of (a) spin up and (b) spin down polarized neutrons at $T=473 \mathrm{~K}$ and at different hydrogen pressures. The count rate is normalized to monitor and the data for different pressures are vertically offset by 0.05 for clarity. The critical edge $Q_{c}$ at $Q_{z} \leqslant 0.017 \AA^{-1}$, which is the upper limit of the total external reflection region, is shown as a black vertical line. The resonances, marked $m=1-4$, are indicated by black dashed lines. Fits over the extended region are marked by black lines. 
TABLE II. Parameters from the fit of the extended and pre-edge regions. Uncertainties are estimated of the order of $1 \%$ for the thickness values and $10 \%$ for the $\mathrm{SLD}_{\text {real }}$ and $\mathrm{SLD}_{\mathrm{im}}$ values.

\begin{tabular}{|c|c|c|c|}
\hline \multicolumn{4}{|c|}{ Extended region } \\
\hline $\begin{array}{l}\text { Pressure } \\
\text { (mbar) }\end{array}$ & $\begin{array}{c}\text { Thickness } \\
(\AA)\end{array}$ & $\begin{array}{c}\mathrm{SLD}_{\text {real }} \\
\left(10^{-6} \AA^{-2}\right)\end{array}$ & $\begin{array}{c}\mathrm{SLD}_{\mathrm{im}} \\
\left(10^{-8} \AA^{-2}\right)\end{array}$ \\
\hline$\sim 2.0 \times 10^{-6}$ & 1071(11) & $-0.32(4)$ & $0.44(5)$ \\
\hline $0.42(5)$ & $1077(11)$ & $-0.39(4)$ & $0.43(5)$ \\
\hline $0.77(5)$ & $1076(11)$ & $-0.45(5)$ & $0.45(5)$ \\
\hline $1.3(1)$ & $1080(11)$ & $-0.51(6)$ & $0.48(5)$ \\
\hline $2.0(1)$ & $1084(11)$ & $-0.54(6)$ & $0.52(6)$ \\
\hline $3.2(1)$ & $1087(11)$ & $-0.61(7)$ & $0.55(6)$ \\
\hline $4.5(1)$ & 1091(11) & $-0.66(7)$ & $0.55(6)$ \\
\hline $7.0(2)$ & 1094(11) & $-0.75(8)$ & $0.60(6)$ \\
\hline $14.0(5)$ & $1100(11)$ & $-0.80(8)$ & $0.59(6)$ \\
\hline $26(1)$ & $1103(12)$ & $-0.87(9)$ & $0.64(7)$ \\
\hline$\sim 200$ & $1170(12)$ & $-1.91(20)$ & $0.91(10)$ \\
\hline$\sim 500$ & $1171(12)$ & $-1.98(20)$ & $0.94(10)$ \\
\hline \multicolumn{4}{|c|}{ Pre-edge region } \\
\hline $\begin{array}{l}\text { Pressure } \\
\text { (mbar) }\end{array}$ & $\begin{array}{c}\text { Thickness } \\
(\AA)\end{array}$ & $\begin{array}{c}\operatorname{SLD}_{\text {real }} \\
\left(10^{-6} \AA^{-2}\right)\end{array}$ & $\begin{array}{c}\mathrm{SLD}_{\mathrm{im}} \\
\left(10^{-8} \AA^{-2}\right)\end{array}$ \\
\hline$\sim 2.0 \times 10^{-6}$ & $1067(11)$ & $-0.36(4)$ & $0.43(5)$ \\
\hline $0.42(5)$ & 1074(11) & $-0.44(5)$ & $0.44(5)$ \\
\hline $0.77(5)$ & 1081(11) & $-0.45(5)$ & $0.42(5)$ \\
\hline $1.3(1)$ & $1085(11)$ & $-0.52(6)$ & $0.47(5)$ \\
\hline $2.0(1)$ & 1091(11) & $-0.53(6)$ & $0.49(5)$ \\
\hline $3.2(1)$ & $1092(11)$ & $-0.62(7)$ & $0.49(5)$ \\
\hline $4.5(1)$ & 1094(11) & $-0.67(7)$ & $0.49(5)$ \\
\hline $7.0(2)$ & $1096(11)$ & $-0.74(8)$ & $0.54(6)$ \\
\hline $14.0(5)$ & $1106(12)$ & $-0.80(8)$ & $0.54(6)$ \\
\hline $26(1)$ & $1110(12)$ & $-0.85(9)$ & $0.59(6)$ \\
\hline$\sim 200$ & $1171(12)$ & $-1.90(20)$ & $0.87(9)$ \\
\hline$\sim 500$ & $1170(12)$ & $-1.99(20)$ & $0.92(10)$ \\
\hline
\end{tabular}

summarized in Table II. The values of the thickness of the $\mathrm{VH}_{x}$ layer from the fit of the pre-edge and extended regions are in good agreement, with an average difference of less than $1 \%$, and agree well with the value reported earlier and extracted from a much larger $Q$ range [31]. The values extracted for $\mathrm{SLD}_{\text {real }}$ for the $\mathrm{VH}_{x}$ layer are consistent within about $2 \%$ between the pre-edge and extended regions. Moreover, the SLD values associated to the lowest data point, $-0.32(4) \times$ $10^{-6} \AA^{-2}$ and $-0.36(4) \times 10^{-6} \AA^{-2}$ from the extended and pre-edge regions, respectively, are very close to the calculated one, $\mathrm{SLD}_{\mathrm{coh}}=-0.319 \times 10^{-6} \AA^{-2}$, assuming a mass density of pure vanadium of $6.08 \mathrm{~g} \mathrm{~cm}^{-3}$ and tabulated values of the bound coherent scattering length. Note that the residual stress in the thin vanadium film results in lattice distortion and slight deviations of the density value. The values of the imaginary part of the SLD, $S_{L D}$ im are in average about $6 \%$ lower for the fits of the pre-edge region than of the extended region, which can be rationalized as an artifact of the fitting procedure due to the insufficient number of degrees of freedom. Indeed, a full reflectivity is necessary to accurately obtain all the parameters that characterize the film by fitting, as we performed previously [31]. However, note that the differences between the results of the fit of the pre-edge and extended regions are small compared to the standard errors of the fitted parameters and that these standard errors are sufficiently small compared to the variation of the fitted parameters for the purpose of this experiment. It follows that, given the available degree of precision, the information on the real and imaginary part of the SLD and the thickness of the vanadium hydride layer are essentially identical when extracted from the pre-edge and extended regions of the reflectivity profiles.

\section{DISCUSSION}

\section{A. Hydrogen concentration extracted from the coherent SLD}

The coherent $\mathrm{SLD}_{\text {coh }}$ of the compound sample $\mathrm{VH}_{x}$ can be calculated from the number density $n_{\mathrm{VH} x}$ and the coherent scattering lengths according to:

$$
\mathrm{SLD}_{\mathrm{coh}}=n_{\mathrm{VH} x}\left[b_{\mathrm{coh}, \mathrm{V}}+x b_{\mathrm{coh}, \mathrm{H}}\right],
$$

where $b_{\text {coh,V }}$ and $b_{\text {coh,H }}$ are the coherent scattering lengths of $\mathrm{V}$ and $\mathrm{H}$, with values of $-0.443 \mathrm{fm}$ and -3.7409 fm, respectively. The number density $n_{\mathrm{VH} x}$ depends on the Avogadro constant $N_{A}$, the molar mass of the compound $M_{\mathrm{VH} x}=M_{\mathrm{V}}+x M_{\mathrm{H}}$, with $M_{\mathrm{V}}=50.9415 \mathrm{~g} \mathrm{~mol}^{-1}$ and $M_{\mathrm{H}}=$ $1.00784 \mathrm{~g} \mathrm{~mol}^{-1}$, and the density of the compound $\rho_{\mathrm{VH} x}$ according to:

$$
n_{\mathrm{VH} x}=\frac{N_{A} \rho_{\mathrm{VH} x}}{M_{\mathrm{VH} x}} .
$$

The compound density $\rho_{\mathrm{VH} x}$ can be estimated from the density of pure $\mathrm{V}, \rho_{\mathrm{V}}$, considering the volume expansion upon hydrogen uptake. The density of the clamped vanadium of $\rho_{\mathrm{V}}=6.308 \mathrm{~g} \mathrm{~cm}^{-3}$ is calculated from published values of the lattice parameters in iron-vanadium superlattices [38]. Further, we assume a linear lattice expansion with an expansion coefficient of $\alpha \approx 0.145$ upon loading with hydrogen (see Sec. IV E). We get:

$$
\rho_{\mathrm{VH} x}=\rho_{\mathrm{V}}\left(\frac{1+x \frac{M_{\mathrm{H}}}{M_{\mathrm{V}}}}{1+x \alpha}\right)
$$

which results in $\rho \approx 5.94 \mathrm{~g} \mathrm{~cm}^{-3}$ for $x=0.5$. It follows that Eq. (10) simplifies to:

$$
\mathrm{SLD}_{\mathrm{coh}}=\frac{n_{\mathrm{V}}\left[b_{\mathrm{coh}, \mathrm{V}}+x b_{\mathrm{coh}, \mathrm{H}}\right]}{1+x \alpha} .
$$

The dependency of $\mathrm{SLD}_{\text {coh }}$ with respect to hydrogen content $x$ is shown in Fig. 5. The relationship is almost linear with $x$ in the interval $x=0-0.5$ and may be approximated by a linear function with the limits of $\operatorname{SLD}_{\text {coh }}(\mathrm{V})=-0.330 \times$ $10^{-6} \AA^{-2}$ and $\operatorname{SLD}_{\text {coh }}\left(\mathrm{VH}_{0.5}\right)=-1.609 \times 10^{-6} \AA^{-2}$. Moreover, the effect of expansion is minor as seen by the dashed line in Fig. 5, and the change in SLD between $x=0$ and 0.5 is almost directly proportional to the composition of the layer. Note, the expansion is only about $7 \%$ (from $\alpha \approx 0.145$ ) and the shift in the $Q_{z}$ positions of the resonances [cf. Eq. (6)] is mainly determined by the change in composition of the $\mathrm{VH}_{x}$ layer. 


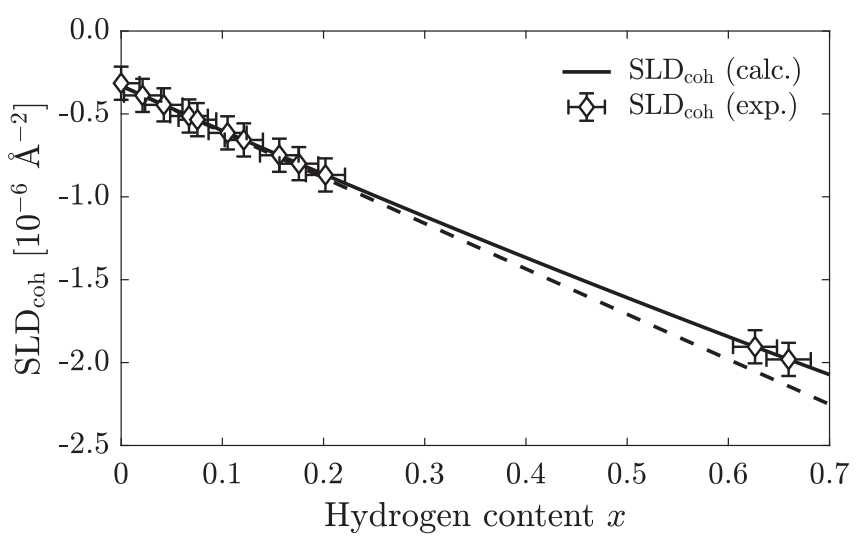

FIG. 5. Coherent SLD (line) plotted versus hydrogen content according to Eq. (13). The experimental values of the SLD from the fit of the extended region are indicated by markers. The dashed line shows SLD values if the volume expansion is neglected.

\section{B. Hydrogen concentration extracted from the attenuation coefficient}

According to the optical theorem [48], the attenuation of the scattering intensity is linked to the complex nature of the scattering process according to

$$
\sigma_{t}=\left(\frac{4 \pi}{k}\right) F^{\prime \prime},
$$

where $\sigma_{t}$ is the total collision cross section per nucleus, i.e., the cross section associated to the attenuation process, and $F^{\prime \prime}$ is the imaginary part of the forward scattering amplitude per nucleus, $F=F^{\prime}+i F^{\prime \prime}$. $F^{\prime \prime}$ contains two terms: The first is associated to the complex part of the SLD, $b^{\prime \prime}$ from $b=$ $b^{\prime}-i b^{\prime \prime}$, which itself is linked to the absorption cross section through $b^{\prime \prime}=\sigma_{\text {abs }} /(2 \lambda)$, and the other is a term associated to the diffuse scattering and involves $S(q)$, the static structure factor. Based on the same considerations as in Sec. IID for the missing signal at the resonances, we consider that the sources of diffuse scattering, in the $Q$-range considered, are the isotropic incoherent scattering and, potentially, SANS or off-specular scattering. The full expression of $F^{\prime \prime}$ becomes:

$$
F^{\prime \prime}=b^{\prime \prime}+k b_{\text {inc }}^{2}+k b_{\text {lowQ }}^{\prime 2} .
$$

Combining Eqs. (14) and (15), the total attenuation cross section for the compound sample $\mathrm{VH}_{x}$ is:

$$
\Sigma_{t} \approx \Sigma_{\mathrm{abs}}+\Sigma_{\mathrm{inc}}+\Sigma_{\mathrm{lowQ}}
$$

The cross section associated to low- $Q$ scattering, $\Sigma_{\text {lowQ }}$, has to be treated as a variable in our analysis. The incoherent and absorption cross sections for the compound, $\Sigma_{\text {inc }}$ and $\Sigma_{\text {abs, }}$, are calculated from the tabulated nuclear cross sections according to:

$$
\begin{gathered}
\Sigma_{\mathrm{inc}}=\frac{n_{V}\left[\sigma_{\mathrm{inc}, \mathrm{V}}+x \sigma_{\mathrm{inc}, \mathrm{H}}\right]}{1+x \alpha}, \\
\Sigma_{\mathrm{abs}}=\frac{n_{V}\left[\sigma_{\mathrm{abs}, \mathrm{V}}+x \sigma_{\mathrm{abs}, \mathrm{H}}\right]}{1+x \alpha} \frac{\lambda}{\lambda_{0}},
\end{gathered}
$$

with $\sigma_{\mathrm{inc}, \mathrm{V}}=5.08$ barn and $\sigma_{\mathrm{inc}, \mathrm{H}}=80.26$ barn, and $\sigma_{\mathrm{abs}, \mathrm{V}}=$ 5.08 barn and $\sigma_{\mathrm{abs}, \mathrm{H}}=0.3326$ barn at $\lambda_{0}=1.798 \AA$ [49]. The

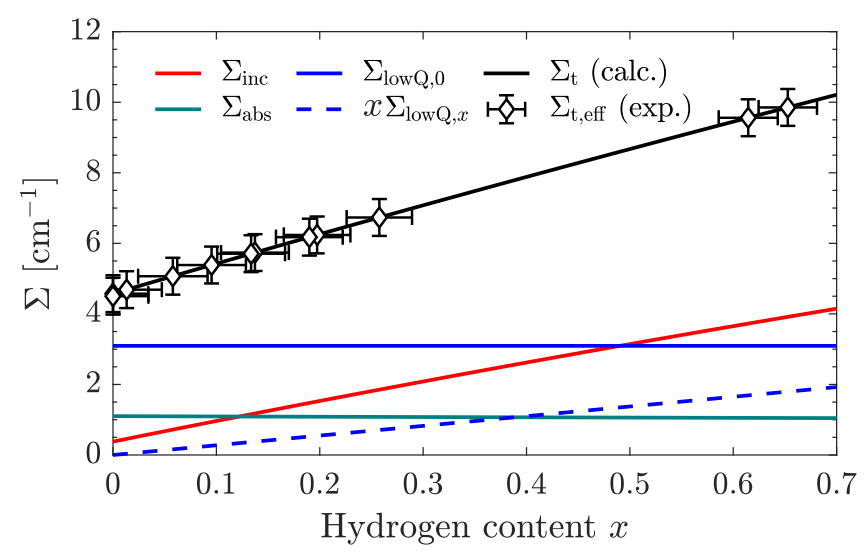

FIG. 6. Cross sections plotted versus hydrogen content: incoherent $\Sigma_{\text {inc }}$ (red line), absorption $\Sigma_{\text {abs }}$ (green line), $x$-independent low- $Q$ scattering $\Sigma_{\text {lowQ, } 0}$ (blue line), $x$-dependent low- $Q$ scattering with $\Sigma_{\text {lowQ,x }}=2.75 \mathrm{~cm}^{-1}$ (dashed blue line), and the sum of these four cross sections $\Sigma_{\mathrm{t}}$ (black line). The experimental values of the attenuation cross section from the fit of the extended region $\Sigma_{\mathrm{t}, \mathrm{eff}}$ are indicated by black markers.

dependencies of $\Sigma_{\text {inc }}$ and $\Sigma_{\text {abs }}$ with the hydrogen concentration are shown as red and green lines, respectively, in Fig. 6.

The fitting procedure defines the attenuation entirely by the imaginary part of the SLD and links it to the absorption cross section [46,47], with $\mathrm{SLD}_{\mathrm{im}}=n_{\mathrm{VH} x} b_{\text {eff }}^{\prime \prime}$, which leads to

$$
\Sigma_{\mathrm{t}, \mathrm{eff}}=2 \lambda \mathrm{SLD}_{\mathrm{im}} .
$$

Using Eqs. (19) and (16), the $S L D_{\text {im }}$ can be extracted for each value of $x$ :

$$
\Sigma_{\mathrm{t}, \mathrm{eff}}=\Sigma_{\mathrm{abs}}+\Sigma_{\mathrm{inc}}+\Sigma_{\text {lowQ }} .
$$

Let us first assume that $\Sigma_{\text {lowQ }}$ is independent of $x$, i.e., the hydrogen does not contribute significantly to the low- $Q$ scattering. Then $\Sigma_{\text {lowQ }}=\Sigma_{\text {lowQ, } 0}$ and Eq. (20) can be solved for $x$ :

$$
x=-\frac{\left(\Sigma_{\mathrm{t}, \mathrm{eff}}-\Sigma_{\mathrm{lowQ}, 0}\right)-n_{V}\left(\sigma_{\mathrm{abs}, \mathrm{V}} \frac{\lambda}{\lambda_{0}}+\sigma_{\mathrm{inc}, \mathrm{V}}\right)}{\alpha\left(\Sigma_{\mathrm{t}, \mathrm{eff}}-\Sigma_{\mathrm{lowQ}, 0}\right)-n_{V}\left(\sigma_{\mathrm{abs}, \mathrm{H}} \frac{\lambda}{\lambda_{0}}+\sigma_{\mathrm{inc}, \mathrm{H}}\right)} .
$$

The value of $\Sigma_{\text {lowQ,0 }}$ is obtained from the lowest pressure data and we get $\Sigma_{\text {t,eff }}=4.57 \mathrm{~cm}^{-1}$ and $\Sigma_{\text {lowQ }, 0}=3.09 \mathrm{~cm}^{-1}$ for the extended region, so $68 \%$ of the attenuation cross section is due to low- $Q$ scattering. For the pre-edge region, we get $\Sigma_{\text {teff }}=4.46 \mathrm{~cm}^{-1}, \Sigma_{\text {lowQ }, 0}=2.98 \mathrm{~cm}^{-1}$, and a ratio of about $67 \%$. Following this approach, the maximum hydrogen content in the film, for the highest-pressure data, is estimated at $x=1.04(12)$ and $x=1.01(12)$ for the extended and pre-edge regions, respectively. These values are significantly above the value of $x=0.66(5)$ extracted from the coherent SLD, which suggests that the hypothesis that $\Sigma_{\text {lowQ }}$ is independent of $x$ is incorrect.

To overcome this discrepancy we may consider instead a linear increase of $\Sigma_{\text {lowQ }}$ with $x: \Sigma_{\text {lowQ }}=\Sigma_{\text {lowQ, } 0}+x \Sigma_{\text {lowQ }, x}$, meaning that a fixed amount of scattering at low $Q$ is independent of $x$ and that a fraction of the scattering at low $Q$ is proportional to $x$. It follows that Eq. (20) expands in a 
quadratic form $A x^{2}+B x+C=0$, with:

$$
\begin{gathered}
A=-\alpha \Sigma_{\text {lowQ }, x}, \\
B=\alpha\left(\Sigma_{\mathrm{t}, \mathrm{eff}}-\Sigma_{\mathrm{lowQ}, 0}\right) \\
-\Sigma_{\text {lowQ }, x}-n_{V}\left(\sigma_{\mathrm{abs}, \mathrm{H}} \frac{\lambda}{\lambda_{0}}+\sigma_{\mathrm{inc}, \mathrm{H}}\right), \\
C=\left(\Sigma_{\mathrm{t}, \mathrm{eff}}-\Sigma_{\mathrm{lowQ}, 0}\right)-n_{V}\left(\sigma_{\mathrm{abs}, \mathrm{V}} \frac{\lambda}{\lambda_{0}}+\sigma_{\mathrm{inc}, \mathrm{V}}\right),
\end{gathered}
$$

with quadratic solutions that do not simplify analytically. The value of $\Sigma_{\text {lowQ,0 }}$ is obtained from the lowest pressure data. As there are two unknown variables, $x$ and $\Sigma_{\text {lowQ, } x}$, the problem cannot be solved without additional information. We may use the maximum hydrogen content, $x=0.65(6)$ and $x=0.63(6)$ for extended and pre-edge regions, respectively, extracted from the analysis of the coherent SLD, which then gives $\Sigma_{\text {lowQ }, x}=2.75 \mathrm{~cm}^{-1}$. The calculated cross sections are shown in Fig. 6 together with the values extracted from the fits.

\section{Low- $Q$ scattering}

The analysis of the attenuation cross section shows that the data cannot be explained without a significant fraction of scattering to low $Q$. This result is consistent with the observation of nonspecular scattering presented in Fig. 3, where we identified a large background that is independent of the hydrogen content, and sharp peaks associated to the enhanced scattering from the vanadium hydride layer at the positions of the resonances.

By analogy, the large background from Fig. 3(b) is associated to the term $\Sigma_{\text {lowQ,0 }}$ of the attenuation cross section. As mentioned in Sec. III, the origin of this $x$-independent, large background could be the blooming effect on the detector, low- $Q$ scattering from the substrate due to overillumination of the thin film, low- $Q$ scattering from the vanadium and iron layers due to lateral heterogeneities, or off-specular magnetic scattering from the iron layers.

Similarly, we associate by analogy the term $\Sigma_{\text {lowQ }, x}$ of the attenuation cross section to the sharp nonspecular peaks in Fig. 3(b). The positions of the peaks in the nonspecular channel is reciprocal to the positions of the dips in the specular channel and, as shown earlier, essentially depends on the SLD of the vanadium hydride layer, thus on the hydrogen content. Similarly, the intensity of the nonspecular peaks depends, partly, on the hydrogen content, as discussed in Eq. (9). However, the intensity also depends on the overillumination through the sample and substrate footprint, which is not simple to account for for nonspecular scattering, and on the amplification factor from the resonance, which depends on the order of the resonance and varies with the incident angle $\theta_{i}$. It follows that, while the presence of nonspecular scattering, which contributes to the attenuation cross section, is confirmed, we cannot dissociate the contributions from the incoherent scattering, from the off-specular scattering, or from potential SANS.

Indeed, at $T=473 \mathrm{~K}$ the $\mathrm{VH}_{x}$ system is in the $\alpha$ phase, or $\alpha^{\prime}$ phase at larger hydrogen content, where the hydrogen is in a liquidlike state [50-53]. However, the temperature is close to the critical temperature of the coexistence of the $(\alpha+\beta)$ and $(\alpha+\varepsilon)$ phases, below which $\mathrm{V}_{2} \mathrm{H}$ precipitates are found [50-53]. It has been shown that such hydride precipitates, as observed in the $\beta$ phase, give rise to SANS [54]. We can rationalize the $x$-dependent low- $Q$ scattering, identified from the attenuation cross section analysis, as SANS originating from the presence of hydride precipitates. While SANS may also contribute to the intensity of the peaks in the nonspecular channel, it could also occur at larger $Q$ values, which correspond to shorter length scales.

The analysis of the attenuation cross section thus provides valuable information on the scattering process at low $Q$ at no extra cost in neutron beam time. While these low- $Q$ scattering processes cannot be distinguished and identified from this method alone, their summed contribution can be accurately quantified. Considering additional sources of information, such as the standard analysis of the SLD, or the distribution of intensity in specular and nonspecular channels by using a PSD, one can evaluate which effects are likely to be at the origin of the missing signal.

In a parametric study such as the one presented here, we have demonstrated that one can distinguish terms in the attenuation cross section based on their dependency with the scanned parameter. A similar measurement could for instance be performed on the hydrogen-free resonator thin film, while varying the applied magnetic field, to quantify the contribution of the off-specular scattering from the magnetic domains of the iron layers to the attenuation cross section. In a similar fashion, for a system where the composition is known, one could quantify the SANS signal as a function of the temperature.

\section{Cross-section measurements}

For the discussion above we have considered the bound scattering cross sections. In particular, the tabulated cross sections used then correspond to the low-temperature response of the $\mathrm{V}$ and $\mathrm{H}$ nuclei. Because of the fairly high temperature of $473 \mathrm{~K}$, the total cross section of $\mathrm{V}$ is increased due to the activation of additional inelastic channels. Conversely, the hydrogen atoms are in their ground state and their inelastic scattering from the lattice dynamics can be neglected. Yet, inelastic scattering from the diffusion motions of $\mathrm{H}$ can be expected as incoherent quasielastic neutron scattering (QENS). The inelastic scattering of $\mathrm{V}$ (lattice dynamics) and $\mathrm{H}$ (QENS) are here ignored and are compensated in the analysis by the terms $\Sigma_{\text {lowQ }, 0}$ for $\mathrm{V}$ and $\Sigma_{\text {lowQ }, x}$ for $\mathrm{H}$.

In a similar fashion, the total hydrogen cross section is dependent on the neutron wavelength. Indeed, at low concentrations hydrogen in vanadium is often described as a lattice gas, which implies that, rather than the bound cross section, the free hydrogen scattering cross section $\sigma_{\text {free }}=\frac{1}{4} \sigma_{\text {bound }}$ might be applied. However, in neutron scattering experiments hydrogen is detected as a particle in an anisotropic harmonic potential $[54,55]$ and is associated to the cross section:

$$
\begin{gathered}
\sigma_{\text {aho }} \approx \sigma_{\text {bound }}\left[1-2 \varepsilon+\frac{32}{15}\left(\varepsilon \varepsilon_{2}+\frac{1}{4} \varepsilon_{1}^{2}\right)\right], \\
\varepsilon_{i}=\frac{E_{n}}{\hbar \omega_{i}}(i=1,2) ; \varepsilon=\frac{1}{3} \varepsilon_{1}+\frac{2}{3} \varepsilon_{2} .
\end{gathered}
$$



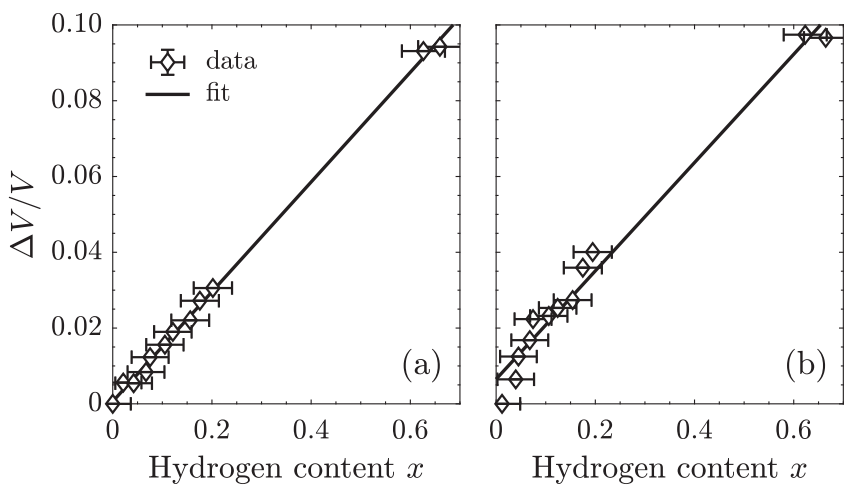

FIG. 7. Relative change in volume $\Delta V / V$ of the $\mathrm{VH}_{x}$ layer plotted as a function of hydrogen content $x$, extracted from the fits of the (a) extended and (b) pre-edge regions. The black line is the fit to the data assuming a linear expansion.

Here $E_{n}$ is the energy of the neutron and $\hbar \omega_{1,2}$ are the energy levels associated with the tetragonal symmetry (hard and soft modes), which are $\hbar \omega_{1}=167(6) \mathrm{meV}$ and $\hbar \omega_{2}=$ 123(6) $\mathrm{meV}$ for hydrogen in $\alpha-\mathrm{VH}_{x}$ [56]. Considering $\lambda=$ $5.225 \AA$, we get an incoherent cross section of $\sigma_{\text {aho }} \approx 0.96 \times$ $\sigma_{\text {inc }}=76.80$ barns, which is very close to the bound value of $\sigma_{\text {bound }}=80.26$ barns.

These two effects of increase of the total cross section of $\mathrm{V}$ and $\mathrm{H}$ due to thermally activated inelastic scattering and decrease of the total cross section of $\mathrm{H}$ (from the bound value) due to its behavior as anisotropic harmonic oscillator, are here minor and cannot explain the missing intensity from the reflected beam, which is attributed in the analysis to low$Q$ scattering. However, would the nonspecular scattering be known and quantified, and because of the high sensitivity of our method to detect any scattering similarly to direct beam measurements, the inelastic scattering cross section, or more generally the total cross section, could be estimated.

\section{E. Expansion coefficients}

Due to the clamping on the $\mathrm{MgO}$ substrate, the change in volume $\Delta V / V$ of the film induced by the hydrogen is restricted to the direction normal to the surface:

$$
\Delta V / V=\Delta L / L \approx \Delta L / L_{0},
$$

where $\Delta L$ is the change in thickness $L$ and $L_{0}$ is the thickness of the pure vanadium film. Figure 7 depicts the relative volume change $\Delta V / V$, obtained from the fit to the reflectivities, plotted as a function of the hydrogen content. Linear expansion coefficients of $\Delta v / \Omega=0.145(2)$ and $0.143(7)$ are found for the extended and pre-edge regions, respectively. Note that these expansion coefficients are based on a model in which the distribution of hydrogen in the vanadium hydride layer is homogeneous.

The expansion coefficients for clamped bulk vanadium are $\Delta v / \Omega=0.18-0.19$ for $\mathrm{H}$ in octahedral sites [57,58] and $0.11-0.135$ for $\mathrm{H}$ in tetrahedral sites [58-64]. The expansion coefficient extracted from our analysis compares well with these values but is slightly larger than the reported values for the hydrogen in tetrahedral sites. This can be rationalized by the possible occupation of some octahedral sites due to the

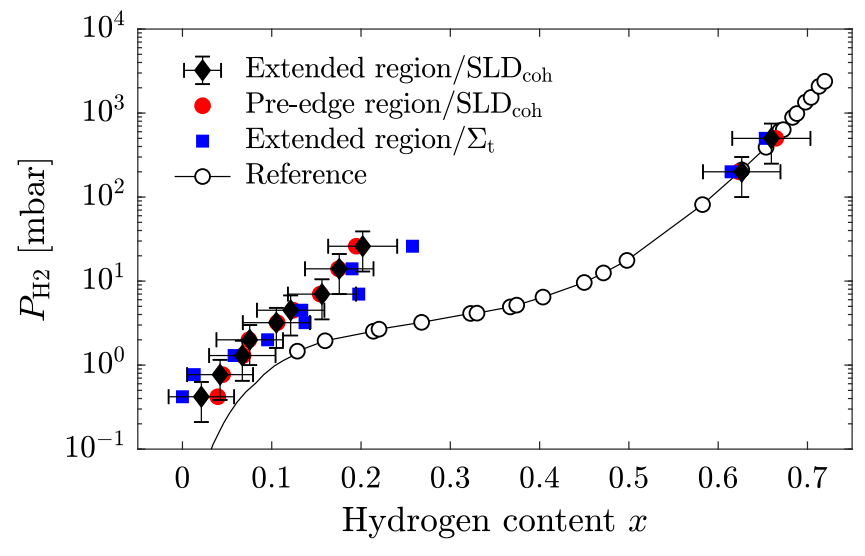

FIG. 8. Isotherm of $\mathrm{VH}_{x}$ based on the hydrogen concentrations determined from the fit of $\mathrm{SLD}_{\mathrm{coh}}$ for the extended region (black diamonds), pre-edge region (red circles), and from the analysis of $\Sigma_{\mathrm{t}}$ for the extended region (blue squares). The pressure axis is in $\log$ scale, and the lowest pressure point for which $x \approx 0$ is not represented. Note that the error bars of two isotherm profiles are omitted for clarity. The black circles and lines represent the isotherm at $473 \mathrm{~K}$ reproduced and adapted from Schober et al. [65].

tetragonal distortion induced by the substrate as well as by the low- $Q$ scattering indicating hydride precipitates. Assuming a mixed occupancy we calculate $64(3) \%$ of the hydrogen atoms occupying tetrahedral sites and 36(3)\% octahedral sites from our expansion coefficient measurement.

\section{F. Isotherms}

The pressure-concentration isotherm based on the hydrogen concentrations determined from $\mathrm{SLD}_{\mathrm{coh}}$ are shown in Fig. 8, for the extended (black diamonds) and pre-edge (red circles) regions. The isotherm extracted from the attenuation cross section $\left(\Sigma_{t}\right)$ for the extended region (blue squares) is also represented. Within the experimental uncertainty the three isotherms are identical, which is a proof-of-concept that consistent information can be obtained by the proposed method.

Note, however, that the presented data are systematically offset in the low-pressure region with respect to previously reported data [65-67]. For comparison the $473 \mathrm{~K}$ isotherm reported in Ref. [65] is reproduced in Fig. 8 as a black line and circles. This systematic offset at low pressure is due to an unforeseen issue with the pressure sensor readings that overestimated the actual pressure in the sample chamber. Unfortunately, the data cannot be properly corrected as the issue cannot be precisely accounted for. However, the high-pressure data, for which the pressure was measured by the pressure regulator of the hydrogen bottle, are in excellent agreement with the reported data.

\section{SUMMARY AND CONCLUSIONS}

In this paper we establish resonant enhancement in a quantum resonator for the study of thin films. We analytically calculate the positions of the resonances, which turn out to be dependent on the thickness and the SLD of the resonator layer. We show that the composition and thickness of a 
resonator film can be extracted reliably from fits to the intensity missing from the totally externally reflected intensity. At this condition, the neutron flux is large as the measurement is an effective direct beam measurement. Moreover, the intensity missing from reflection is related and very sensitive to any attenuation of the beam, may it be absorption or scattering in the resonator layer. This provides a consistency check for the determined composition of the film. Moreover, the method is sensitive to any scattering and allows the indirect detection of small angle scattering as well as potential changes in the neutron scattering cross sections due to the dynamics in the film.

As a model system we study a vanadium hydride resonator thin film. We show that the SLD and the thickness of the layer can be reliably extracted from fits to the position and depth of the dips in the region of total external reflection. We demonstrate that the evaluation of the attenuation cross section provides a very good consistency check for the hydrogen concentrations in the film extracted from the SLD values. From the results we construct pressure-concentration isotherms of vanadium hydride at $T=473 \mathrm{~K}$. In addition, we determine the expansion coefficients of the resonator layer under hydrogen loading indicating a mixed site occupancy above the critical point, which is in line with earlier findings. Moreover, the consistency checks of the beam attenuation allow us to identify additional scattering to low $Q$, which is hard to measure directly. We relate this scattering to small angle scattering emerging from hydride precipitates, which are expected for temperature close to the critical one. We have shown that equivalent information can be obtained from the analysis of the SLD, from the analysis of the dips in the region of total external reflection only, and from the attenuation cross section, which is thus a proof-of-concept of the presented method.
Our method has huge potential to trace changes in composition and thickness of layers as a function of external parameters, like temperature, gas exposure, or magnetic field, since it allows fast, time-resolved, in situ studies. The short measurement times are also beneficial for experiments requiring data on different sample positions or orientations as required for imaging or tomography of inhomogeneous in buried interfaces in thin films [68]. Moreover, the enhancement of scattering allows to detect small angle or inelastic scattering and is another step towards the study of dynamics in thin films with neutrons. The example of vanadium hydride demonstrates the potential of the method but resonators may be constructed for many systems making our approach widely applicable. Materials with elements with a negative SLD, other than vanadium, are ideal to form good resonators. Examples are lithium or hydrogen, which are of great interest for energy research, soft matter physics, pharmacy, and biology. For instance, a lipid membrane confined between sapphire and heavy water may result in a resonating wave field, which should allow the study of membrane dynamics as well as diffusion into and through membranes.

Access to the raw experimental neutron data is provided according to the ILL data policies [69].

\section{ACKNOWLEDGMENTS}

The authors thank the Swedish Research Council for funding under Grant No. 2016-06958 as well as for the RFI funding of the national infrastructure Super ADAM (Grant No. 2015-05988). The authors thank the Institut LaueLangevin for access to neutron beam facilities.
[1] F. J. Giessibl, Advances in atomic force microscopy, Rev. Mod. Phys. 75, 949 (2003).

[2] R. F. Egerton (ed.), Physical Principles of Electron Microscopy (Springer International Publishing, Switzerland, 2016).

[3] S. Hüfner (ed.), Photoelectron Spectroscopy (Springer-Verlag, Berlin, Heidelberg, 2003).

[4] Y. Yoneda, Anomalous surface reflection of X rays, Phys. Rev. 131, 2010 (1963).

[5] J. Wang, M. J. Bedzyk, and M. Caffrey, Resonance-enhanced xrays in thin films: A structure probe for membranes and surface layers, Science 258, 775 (1992).

[6] V. Holý and T. Baumbach, Nonspecular x-ray reflection from rough multilayers, Phys. Rev. B 49, 10668 (1994).

[7] S. K. Sinha, E. B. Sirota, S. Garoff, and H. B. Stanley, X-ray and neutron scattering from rough surfaces, Phys. Rev. B 38, 2297 (1988).

[8] S. K. Sinha, Surface roughness by X-ray and neutron scattering methods, Acta Phys. Pol. A 89, 219 (1996).

[9] U. Klemradt, M. Fromm, G. Landmesser, H. Amschler, and J. Peisl, Surface effects in displacive phase transformations studied by X-ray specular reflectivity, Physica B 248, 83 (1998).

[10] M. Tolan, G. König, L. Brügemann, W. Press, F. Brinkop, and J. P. Kotthaus, X-ray diffraction from laterally structured surfaces: Total external reflection and grating truncation rods, Europhys. Lett. 20, 223 (1992).
[11] S. Kalinin, N. Balke, S. Jesse, A. Tselev, A. Kumar, T. M. Arruda, S. Guo, and R. Proksch, Li-ion dynamics and reactivity on the nanoscale, Mater. Today 14, 548 (2011).

[12] M. R. Karim, K. Hatakeyama, T. Matsui, H. Takehira, T. Taniguchi, M. Koinuma, Y. Matsumoto, T. Akutagawa, T. Nakamura, S.-I. Noro, T. Yamada, H. Kitagawa, and S. Hayami, Graphene oxide nanosheet with high proton conductivity, J. Am. Chem. Soc. 135, 8097 (2013).

[13] A. Royant, K. Edman, T. Ursby, E. Pebay-Peyroula, E. M. Landau, and R. Neutze, Helix deformation is coupled to vectorial proton transport in the photocycle of bacteriorhodopsin, Nature (London) 406, 645 (2000).

[14] K. Murzyn, W. Zhao, M. Karttunen, M. Kurdziel, and T. Róg, Dynamics of water at membrane surfaces: Effect of headgroup structure, Biointerphases 1, 98 (2006).

[15] B. Bagchi, Water dynamics in the hydration layer around proteins and micelles, Chem. Rev. 105, 3197 (2005).

[16] Z. Fakhraai and J. A. Forrest, Measuring the surface dynamics of glassy polymers, Science 319, 600 (2008).

[17] M. Tress, E. U. Mapesa, W. Kossack, W. K. Kipnusu, M. Reiche, and F. Kremer, Glassy dynamics in condensed isolated polymer chains, Science 341, 1371 (2013).

[18] R. D. Priestley, C. J. Ellison, L. J. Broadbelt, and J. M. Torkelson, Structural relaxation of polymer glasses at surfaces, interfaces, and in between, Science 309, 456 (2005). 
[19] M. Wolff and P. Gutfreund, Neutron reflectivity for the investigation of coatings and functional layers, in Handbook of Modern Coating Technologies, Advanced Characterisation Methods, edited by M. Aliofkhazraei, A. Nasar, M. Chipara, N. Laidani, and J. T. M. De Hosson (Elsevier, Amsterdam, 2021), pp. 143-175.

[20] H. Fritzsche, F. Klose, C. Rehm, Z. Tun, M. Wolff, and B. Hjörvarsson, Neutron reflectometry, in Neutron Scattering and Other Nuclear Techniques for Hydrogen in Materials, edited by H. Fritzsche, J. Huot, and D. Fruchart (Springer International Publishing, 2016), pp. 115-158.

[21] M. Wolff, Grazing incidence scattering, EPJ Web Conf. 188, 04002 (2018).

[22] F. Pfeiffer, V. Leiner, P. Høghøj, and I. Anderson, Submicrometer Coherent Neutron Beam Production Using a Thin-Film Waveguide, Phys. Rev. Lett. 88, 055507 (2002).

[23] F. Radu and V. K. Ignatovich, Theoretical description of neutron resonances in multilayer systems, Physica B 292, 160 (2000).

[24] H. Zhang, P. D. Gallagher, S. K. Satija, R. M. Lindstrom, R. L. Paul, T. P. Russell, P. Lambooy, and E. J. Kramer, Grazing Incidence Prompt Gamma Emissions and Resonance-enhanced Neutron Standing Waves in a Thin Film, Phys. Rev. Lett. 72, 3044 (1994).

[25] V. L. Aksenov, Yu. V. Nikitenko, F. Radu, Yu. M. Gledenov, and P. V. Sedyshev, Observation of resonance enhanced neutron standing waves through $(\mathrm{n}, \alpha)$ reaction, Physica B 276-278, 946 (2000)

[26] H. Zhang, S. Satija, P. Gallagher, J. Dura, K. Ritley, C. Flynn, and J. Ankner, Diffraction of neutron standing waves in thin films with resonance enhancement, Physica B 221, 450 (1996).

[27] F. Radu, V. Leiner, K. Westerholt, H. Zabel, J. McCord, A. Vorobiev, J. Major, D. Jullien, H. Humblot, and F. Tasset, Magnetic induction and domain walls in magnetic thin films at remanence, J. Phys. Condens. Matter 17, 1711 (2005).

[28] Y. N. Khaidukov and Y. V. Nikitenko, Magnetic non-collinear neutron wave resonator, Nucl. Instrum. Methods Phys. Res., Sect. A 629, 245 (2011).

[29] Y. N. Khaidukov, B. Nagy, J.-H. Kim, T. Keller, A. Rühm, Y. V. Nikitenko, K. N. Zhernenkov, J. Stahn, L. F. Kiss, A. Cisk, L. Bottyán, and V. L. Aksenov, On the feasibility to study inverse proximity effect in a single S/F bilayer by Polarised Neutron Reflectometry, JETP Lett. 98, 107 (2013).

[30] S. V. Kozhevnikov, Planar neutron waveguides, Phys. Part. Nuclei 50, 300 (2019).

[31] M. Wolff, A. Devishvili, J. A. Dura, F. A. Adlmann, B. Kitchen, G. K. Pálsson, H. Palonen, B. B. Maranville, C. F. Majkrzak, and B. P. Toperverg, Nuclear Spin Incoherent Neutron Scattering from Quantum Well Resonators, Phys. Rev. Lett. 123, 016101 (2019).

[32] S. Jaksch, O. Holderer, M. Gvaramia, M. Ohl, M. Monkenbusch, and H. Frielinghaus, Nanoscale rheology at solid-complex fluid interfaces, Sci. Rep. 7, 4417 (2017).

[33] H. Frielinghaus, M. Gvaramia, G. Mangiapia, S. Jaksch, M. Ganeva, A. Koutsioubas, S. Mattauch, M. Ohl, M. Monkenbusch, and O. Holderer, New tools for grazing incidence neutron scattering experiments open perspectives to study nano-scale tribology mechanisms, Nucl. Instrum. Methods Phys. Res., Sect. A 871, 72 (2017).
[34] J. Rowe, K. Sköld, H. Flotow, and J. Rush, Quasielastic neutron scattering by hydrogen in the $\alpha$ and $\beta$ phases of vanadium hydride, J. Phys. Chem. Solids 32, 41 (1971).

[35] X. Xin, G. K. Pálsson, M. Wolff, and B. Hjörvarsson, FiniteSize Effects: Hydrogen in Fe/V(001) Superlattices, Phys. Rev. Lett. 113, 046103 (2014).

[36] L. Mooij, W. Huang, S. A. Droulias, R. Johansson, O. Hartmann, X. Xin, H. Palonen, R. H. Scheicher, M. Wolff, and B. Hjörvarsson, Influence of site occupancy on diffusion of hydrogen in vanadium, Phys. Rev. B 95, 064310 (2017).

[37] S. A. Holt, A. P. Le Brun, C. F. Majkrzak, D. J. McGillivray, F. Heinrich, M. Lösche, and J. H. Lakey, An ion-channelcontaining model membrane: Structural determination by magnetic contrast neutron reflectometry, Soft Matter 5, 2576 (2009).

[38] S. A. Droulias, G. K. Pálsson, H. Palonen, A. Hasan, K. Leifer, V. Kapaklis, B. Hjörvarsson, and M. Wolff, Crystal perfection by strain engineering: The case of Fe/V (001), Thin Solid Films 636, 608 (2017).

[39] P. Isberg, B. Hjörvarsson, R. Wäppling, E. B. Svedberg, and L. Hultman, Growth of epitaxial FeV (001) superlattice films, Vacuum 48, 483 (1997).

[40] H. Bleichert and H. Wenzl, Die unterschiedliche Löslichkeit der Wasserstoffisotope Protium, Deuterium und Tritium in Vanadium, Phys. Stat. Sol. (b) 144, 361 (1987).

[41] R. Griessen and T. Riesterer, Heat of formation models, in $H y$ drogen in Intermetallic Compounds I, edited by L. Schlapbach (Springer-Verlag, Berlin, Heidelberg, 1988), pp. 219-284.

[42] M. Nagano, Y. Hayashi, N. Ohtani, M. Isshiki, and K. Igaki, Hydrogen diffusivity in high purity alpha iron, Scr. Metall. 16, 973 (1982).

[43] A. Vorobiev, A. Devishvilli, G. Pálsson, H. Rundlöf, N. Johansson, A. Olsson, A. Dennison, M. Wolff, B. Giroud, O. Aguettaz, and B. Hjörvarsson, Recent upgrade of the polarized neutron reflectometer Super ADAM, Neutron News 26, 25 (2015).

[44] A. Devishvili, K. Zhernenkov, A. J. C. Dennison, B. P. Toperverg, M. Wolff, B. Hjörvarsson, and H. Zabel, SuperADAM: Upgraded polarized neutron reflectometer at the Institut Laue-Langevin, Rev. Sci. Instrum. 84, 025112 (2013).

[45] B. P. Toperverg, Polarized neutron reflectometry of magnetic nanostructures, Phys. Metals Metallogr. 116, 1337 (2015).

[46] B. P. Toperverg and V. Derizlazov, Softwares for fitting of specular reflection and simulations of off-specular scattering (unpublished). Available on the instrument site or from Boris. Toperverg@ rub.de.

[47] A. Klechikov, BoToFit, Neutron Reflectivity fitting software (unpublished). Available on https://github.com/AlexeyKlechikov/BoToFit.

[48] V. F. Sears, Schrödinger equation for neutron optics, Physica B+C 151, 156 (1988).

[49] V. F. Sears, Neutron scattering lengths and cross sections, Neutron News 3, 26 (1992).

[50] G. Alefeld, Wasserstoff in metallen als beispiel für ein gittergas mit phasenumwandlungen, Phys. Stat. Sol. (b) 32, 67 (1969).

[51] T. Schober and A. Carl, A differential thermal analysis study of the vanadium-hydrogen system, Phys. Stat. Sol. (a) 43, 443 (1977). 
[52] W. Pesch, T. Schober, and H. Wenzl, A TEM study of the phase diagrams VH and VD, Scr. Metall. 16, 307 (1982).

[53] R. Lässer, Properties of protium, deuterium and tritium in selected metals, in Tritium and Helium-3 in Metals, Vol. 9 (Springer-Verlag, Berlin, Heidelberg, 1989).

[54] A. Miksovsky, H. Amenitsch, H. Rauch, and E. Seidl, Ultra small-angle scattering of neutrons from vanadium-hydrogen systems, Phys. Stat. Sol. (a) 130, 365 (1992).

[55] G. Steyrer and J. Peisl, On the total cross-section for thermal neutrons of hydrogen in niobium, Z. Phys. B Condens. Matter 64, 425 (1986).

[56] G. Verdan, R. Rubin, and W. Kley, The dynamics of hydrogen impurities in niobium and vanadium, in Neutron Inelastic Scattering, Vol. 1 (International Atomic Energy Agency, Vienna, 1968), pp. 223-231.

[57] Y. Fukai, State of hydrogen in BCC metals: Its quantummechanical character, Jpn. J. Appl. Phys. 22, 207 (1983).

[58] G. K. Pálsson, M. Wälde, M. Amft, Y. Wu, M. Ahlberg, M. Wolff, A. Pundt, and B. Hjörvarsson, Hydrogen site occupancy and strength of forces in nanosized metal hydrides, Phys. Rev. B 85, 195407 (2012).

[59] A. J. Maeland, Investigation of the Vanadium-Hydrogen system by X-ray diffraction techniques, J. Phys. Chem. 68, 2197 (1964).

[60] G. Schaumann, J. Völki, and G. Alefeld, The diffusion coefficients of hydrogen and deuterium in vanadium, niobium, and tantalum by gorsky-effect measurements, Phys. Stat. Sol. (b) 42, 401 (1970).
[61] A. Magerl, B. Berre, and G. Alefeld, Changes of the elastic constants of $\mathrm{V}, \mathrm{Nb}$, and $\mathrm{Ta}$ by hydrogen and deuterium, Phys. Stat. Sol. (a) 36, 161 (1976).

[62] T. Schober, C. Dieker, and R. Feenstra, Dilute hydrides, deuterides and tritides of $\mathrm{V}, \mathrm{Nb}$ and Ta: Density measurements, J. Phys. F: Met. Phys. 18, 1119 (1988).

[63] G. Andersson, B. Hjörvarsson, and H. Zabel, Hydrogeninduced lattice expansion of vanadium in a $\mathrm{Fe} / \mathrm{V}$ (001) single-crystal superlattice, Phys. Rev. B 55, 15905 (1997).

[64] R. Johansson, R. Ahuja, O. Eriksson, B. Hjörvarsson, and R. H. Scheicher, Effect of uniaxial strain on the site occupancy of hydrogen in vanadium from density-functional calculations, Sci. Rep. 5, 10301 (2015).

[65] T. Schober, Vanadium-, Niobium- and Tantalum-Hydrogen, in Hydrogen Metal Systems I, Vol. 49 (Trans Tech Publications Ltd, Switzerland, 1996), pp. 357-422.

[66] P. Meuffels, Hydrogen and deuterium in vanadium as lattice gases with repulsive short-range interactions, KFA-Jülich Report \#2081 (1986).

[67] K. Fujita, K. Huang, and M. Tada, Study on equilibrium of TaH, Nb-H, V-H system, J. Jpn. Inst. Met. 43, 601 (1979).

[68] K. Sakurai, J. Jiang, M. Mizusawa, T. Ito, K. Akutsu, and N. Miyata, Neutron visualisation of inhomogeneous buried interfaces in thin films, Sci. Rep. 9, 571 (2019).

[69] A. Perrichon, A. Devishvili, M. Karlsson, K. Komander, R. Lavén, G. K. Pálsson, A. Vorobiev, and M. Wolff, Experimental data measured on Super ADAM at the Institut Laue-Langevin (2019), doi:10.5291/ILL-DATA.CRG-2600. 